\title{
DIVERSIDADE E BIOMASSA FITOPLANCTÔNICA EM VIVEIRO DE CARCINICULTURA MARINHA NO ESTADO DO PARÁ
}

\author{
Rayette Souza da Silva $1^{1}$ \\ Alex da Silva Lobão de Souza ${ }^{2}$ \\ Glauber David Almeida Palheta ${ }^{3}$ \\ Maria Sintia Monteiro da Costa ${ }^{4}$ \\ Nuno Filipe Alves Correia de Melo ${ }^{5}$
}

\begin{abstract}
RESUMO
Este trabalho objetivou avaliar durante um ciclo de cultivo de Litopenaeus vannamei a comunidade fitoplanctônica e parâmetros abióticos em duas estações dentro de um viveiro em Curuçá (PA), norte do Brasil. Foram medidos transparência, $\mathrm{pH}$, oxigênio dissolvido, salinidade e temperatura. Considerando os últimos quatro parâmetros, foram registrados na água de superfície e próximo ao fundo. Além disso, também foram coletadas amostras para estudar o fiitoplâncton e concentrações de clorofila "a". A temperatura variou de 31,5 a $35^{\circ} \mathrm{C}$; oxigênio dissolvido de 4,2 a 15,5 mg/L; pH entre 8,1 e 9,4; salinidade de 26,9 a $30 \mathrm{ppm}$. A transparência diminuiu de 55 para $17 \mathrm{~cm}$. Clorofila $a$ variou de 2,33 a $471,34 \mathrm{mg} / \mathrm{m}^{3}$. Em relação à análise do fitoplâncton, foram identificados 95 taxa e Bacillariophyta foi o grupo mais representativo, com dominância do Navicula sp, Pleurosigma sp e Nitzschia sp. A maior densidade registrada foi de 104.400 e a menor foi $3.600 \mathrm{org} / \mathrm{L}$. A diversidade média na Estação 01 foi 1,49 bits/ind e na Estação 02, 1,43 bits/ind. Concluiu-se que: a divisão Bacillariophyta foi o grupo mais importante (riqueza e densidade); a classe dinophyceae revelou estar melhor adaptada em águas mais claras; Os valores de Clorofila "a" apresentaram uma relação direta com a alimentação artificial, aumentando com o tempo; Apesar da grande flutuação dos parâmetros abióticos como pH , oxigênio dissolvido e transparência , o sistema de cultivo foi considerado homogêneo.
\end{abstract}

Palavras-Chave: Carcinicultura, Litopenaeus vannamei, Microalgas.

\section{DIVERSITY AND BIOMASS PHYTOPLANKTON IN MARINE SHRIMP FARMING IN PARA STATE}

\begin{abstract}
This work aimed to evaluate the the phytoplanktonic community and the abiotic parameters during a cycle of cultivation of Litopenaeus vannamei in two stations in a pond in Curuçá (PA), northern Brazil. Were measured transparency, $\mathrm{pH}$, dissolved oxygen, salinity and temperature. Considering the last four parameters, were registered in the surface water and the bottom set. Additionally, also were collected samples to study the fitoplankton and chlorophyll "a" concentrations. The temperature values were between 31,5 and $35{ }^{\circ} \mathrm{C}$; dissolved oxygen concentrations were between 4,2 and 15,5 mg/L; $\mathrm{pH}$ between 8,1 and 9,4;

\footnotetext{
${ }^{1}$ Professora do Instituto Federal de Educação, Ciência e Tecnologia do Pará - IFPA. Correspondência.

2 Professor do Curso Técnico de Pesca e Aquicultura do Instituto Federal de Educação Ciência e Tecnologia do Pará (IFPA) Campus Abaetetuba.

${ }^{3}$ Professor Adjunto I da Universidade Federal Rural da Amazônia e pesquisador no Instituto Sócio Ambiental dos Recursos Hídricos (ISARH/UFRA)

${ }^{4}$ Instituto Federal de Educação, Ciência e Tecnologia do Pará - IFPA

5 Professor Associado I da Universidade Federal Rural da Amazônia.
}

Silva RS, Souza ASL, Palheta GDA, Costa MSM, Melo NFAC. Diversidade e biomassa fitoplanctônica em viveiro de carcinicultura marinha no estado do Pará. Vet. e Zootec. 2018 jun.; 25(2): 142-154. 
salinity from 26,9 to $30 \mathrm{ppm}$. The transparency decreased from 55 to $17 \mathrm{~cm}$. The Chlorophyll a concentrations were from 2,33 to $471,34 \mathrm{mg} / \mathrm{m} 3$. The transparency decreased of 55 to 17 $\mathrm{cm}$. Chlorophyll a from 2,33 to $471,34 \mathrm{mg} / \mathrm{m} 3$. Regarding the fitoplankton analyses, were identified 95 taxa and Bacillariophyta was the most representative group, with dominance of the Navicula sp, Pleurosigma sp and Nitzschia sp. The largest density registered was of 104.400 and to smallest was $3.600 \mathrm{org} / \mathrm{L}$. The mean diversity at the station 01 was 1,49 bits/ind and in the station 02 was 1,43 bits/ind. Enter the main conclusions: the fitoplanktonic community was dominated by the diatom and the division Bacillariophyta was the most important group (wealth and density); the class dinophyceae revealed to be better adapted in the clearer waters; Chlorophyll "a" values presented a direct relationship to the feeding artificial, increasing with the time. Despite the great fluctuation of abiotic parameters such $\mathrm{pH}$, dissolved oxygen and transparency, the cultivation system was considered homogeneous. Key-words: Shrimp Farming, Litopenaeus vannamei, Microalgae.

\section{DIVERSIDAD Y BIOMASA DE FITOPLANCTON EN EL CULTIVO DE CAMARÓN MARINO DEL ESTADO DE PARÁ}

\section{RESUMEN}

Este estudio evaluó durante un ciclo de cultivo de Litopenaeus vannamei la comunidad de fitoplancton y los parámetros abióticos en dos estaciones dentro de un cuarto de niños en Curuçá (PA), el norte de Brasil. Se midieron la transparencia, $\mathrm{pH}$, oxígeno disuelto, la salinidad y la temperatura. Teniendo en cuenta los últimos cuatro parámetros se registraron en la superficie del agua y cerca del fondo. Además, también se recogieron muestras para estudiar la concentración fiitoplâncton y clorofila "a". La temperatura varió desde 31,5 hasta $35^{\circ} \mathrm{C}$; Oxígeno disuelto 4,2-15,5 mg / L; pH entre 8,1 y 9,4; la salinidad de 26.9 a 30 ppm. Transparencia redujo de 55 a $17 \mathrm{~cm}$. La clorofila varió desde 2,33 hasta 471,34 mg / m3. En cuanto al análisis de fitoplancton se identificaron 95 tasa Bacillariofitas y fue el grupo más representativo, con predominio de Navivcula sp sp Pleurosigma y Nitzschia sp. La densidad más alta registrada fue de 104.400 y el más bajo fue de 3.600 org / L. La diversidad promedio en la estación 01 era de 1,49 bits / ind y de la estación 02, 1,43 bits / ind. Se concluyó que: la división Bacillariophyta era el grupo más importante (riqueza y densidad); Dinophyceae la clase resultó ser el más adecuado en las aguas más claras; Los valores de clorofila "a" tenían una relación directa con la alimentación artificial, aumentando con el tiempo; A pesar de las grandes fluctuaciones de parámetros abióticos tales como el $\mathrm{pH}$, el oxígeno disuelto y la transparencia, sistema de cultivo se consideró homogénea.

Palabras chave: Cultivo de camarón, Litopenaues vannamei, Microalgas.

\section{INTRODUÇÃO}

A carcinicultura vem se destacando e para continuar crescendo e de forma sustentável é necessário realizar uma avaliação ambiental da área. Em viveiros, as condições da água estão sempre mudando, sendo fundamental observar o comportamento das principais variáveis físicas, químicas e biológicas que possam ser manipuladas. Com isso, é possível conferir aos animais, o maior conforto possível, refletindo positivamente na produtividade do sistema (1).

Nos viveiros de engorda de camarão marinho, o fitoplâncton desempenha um papel ecológico de grande importância como produtor primário, governando os principais processos físicos e químicos deste ecossistema. Dentre os vários processos e aportes no qual o fitoplâncton está envolvido destacam-se: (a) a produção de oxigênio dissolvido através da reação de fotossíntese (1); (b) a assimilação de nutrientes, incluindo a amônia e outros 
metabólitos tóxicos para o camarão, que são seqüestrados da água e convertidos em compostos orgânicos, melhorando os parâmetros dequalidade da água (2), e; (c) o aporte de nutrientes essenciais que funcionam como fonte alimentar indireta para os camarões cultivados (3).

Apesar de estudos que abordem o fitoplâncton e/ou variáveis abióticas em viveiros de carcinicultura marinha estarem sendo desenvolvidos no Brasil (4-10) existe uma escassez de dados no estado do Pará, visto que os trabalhos desenvolvidos em águas estuarinas foram restritos à ambientes naturais (11-17) e não em ambientes de cultivo. Entretanto, sabe-se que conhecer a comunidade fitoplanctônica é imprescindível para o desenvolvimento da carcinicultura, já que é necessária sua caracterização e controle populacional, visando obter estabilidade e um bom desenvolvimento no manejo do sistema, e ao maximizar o uso do alimento natural presente nos viveiros, diminuir os custos com ração.

Desta forma, objetiva-se avaliar os parâmetros abióticos e caracterizar a comunidade fitoplanctônica em termos de riqueza, abundância, frequência de ocorrência e biomassa durante um ciclo de cultivo (97 dias) de Litopenaeus vannamei (Boone, 1931) no município de Curuçá - Pará.

\section{MATERIAL E MÉTODOS}

O empreendimento estudado está instalado no município de Curuçá ( $00^{\circ} 37^{\prime} 30^{\prime}$ 'S e $47^{\circ}$ 52' 30"O) que pertence à Mesorregião do Nordeste Paraense e à Microrregião do salgado Estado do Pará. O município tem precipitações abundantes que ultrapassam os $2.000 \mathrm{~mm}$ anuais, sendo os meses mais chuvosos de janeiro a junho e menos chuvosos de julho a dezembro. Apresenta clima equatorial Amazônico tipo Am da classificação de Kôppen caracterizado pelas temperaturas elevadas e pequena amplitude térmica, com média de $27^{\circ} \mathrm{C}$ (18).

Antes de iniciar o cultivo, o viveiro recebeu $2.000 \mathrm{~kg}$ de calcário e quatro carotes de 6 $\mathrm{kg}$ de cloro para desinfecção. O sistema de cultivo adotado foi semi-intensivo, com troca zero de água, havendo apenas complementação quando necessário. Não foi utilizado nenhum sistema de aeração artificial e também não foram realizados procedimentos de fertilização e calagens de manutenção durante o cultivo. A quantidade estocada foi de 953.000 Pós-Larvas com 20 dias de vida (PL20), correspondendo a uma densidade de 21,8 camarões $/ \mathrm{m}^{2}$.

Até o $25^{\circ}$ dia de cultivo, foi utilizada uma ração comercial farelada com $45 \%$ de proteína, sendo administrada à lanço e somente uma vez ao dia. A partir daí até o final do cultivo, uma ração extrusada com $35 \%$ de proteína bruta passou a ser utilizada, sendo administrada à lanço e na bandeja de alimentação tanto pela manhã quanto pela tarde. A fazenda adotou um sistema de despesca parcelada, sendo realizada ao todo, quatro: 02/12; 10/12; 16/12; e no dia 22 de dezembro de 2008 foi realizada a última despesca, finalizando o ciclo de cultivo, que teve 97 dias.

$\mathrm{O}$ viveiro amostrado é o que possui maior área, 3,5 ha e cerca de 1,5 metro de profundidade média. Para sua amostragem, foram determinadas duas estações de coleta - E1 próximo à saída de água (S $00^{\circ} 41^{\prime} 56,6^{\prime}$ "W 4750'15,0”) e E2 - no meio do viveiro (S $00^{\circ} 41^{\prime} 57,9^{\prime}$ 'W 47 50'16,9”) (Figura 1). A amostragem teve periodicidade quinzenal durante um ciclo de cultivo, de 20/09/08 a 05/12/08.

Foram coletadas amostras de água com o auxílio de uma garrafa de Van Dorn na superfície e próximo ao fundo para realizar medição in situ de: Oxigênio Dissolvido - OD (através de um oxímetro Instrutherm modelo MO-890); Temperatura (leitura de um termistor acoplado à sonda do oxímetro); $\mathrm{pH}$ (através de leitura em um peagômetro Instrutherm modelo 1800); Salinidade (uso de refratômetro Instrutherm modelo RTS-101ATC). A transparência da água foi medida entre 10 e 14 h (19) através do desaparecimento visual do Disco de Secchi com $30 \mathrm{~cm}$ de diâmetro. 


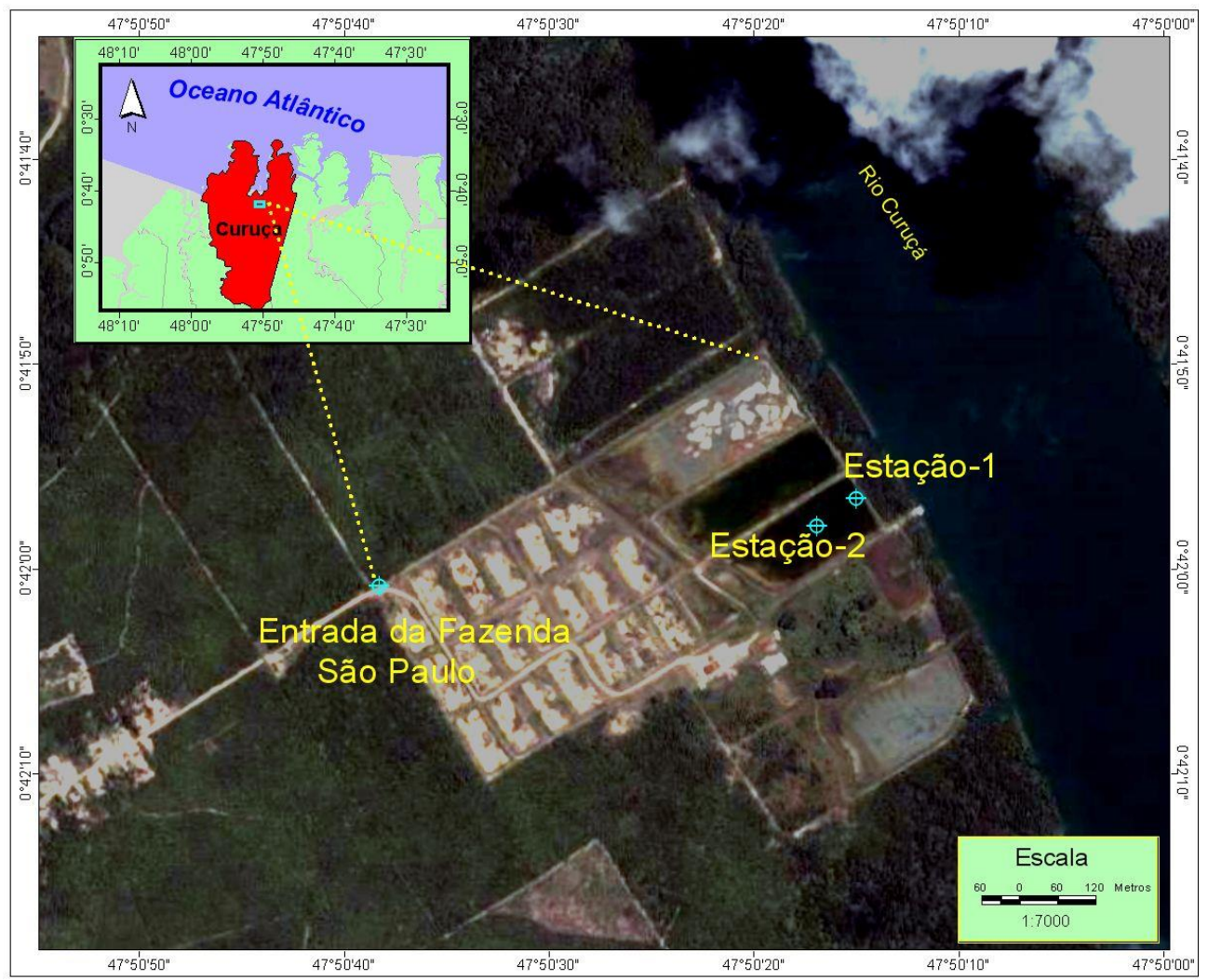

Figura 1. Mapa de localização da fazenda São Paulo, Município de Curuçá - PA, mostrando as duas estações de coleta no viveiro amostrado (Fonte: Insumo de Satélite TerraMetrics - Imagem Google Earth - julho de 2008).

As amostras para a determinação da biomassa fitoplanctônica (clorofila-a) foram obtidas através de coleta direta na subsuperfície da água e fixadas em gelo, com tratamento das amostras segundo recomendação da Unesco (20) e leitura através do espectrofotômetro marca Biospectro SP-22, seguindo o método de Parsons e Strickland (21).

A amostragem qualitativa do fitoplâncton ocorreu com arrasto horizontalmente à subsuperfície com rede cilindro-cônica de $64 \mu \mathrm{m}$ e o material filtrado foi acondicionado em frascos de polietileno (devidamente etiquetados) e fixado com formol neutralizado com tetraborato de sódio até uma concentração final de $4 \%$. Para determinação da densidade foram coletados $100 \mathrm{~mL}$ de amostra, acondicionadas e fixadas da mesma forma.

Para determinação da composição fitoplanctônica foram analisadas no mínimo, cinco lâminas não permanentes sob objetiva de $40 \mathrm{X}$ e $100 \mathrm{X}$, esta com óleo de imersão, A sinopse taxonômica foi baseada, principalmente, na classificação apresentada por Round et al. (22) para as diatomáceas, Steindinger e Tangen (23) para os dinoflagelados, Desikachary (24) para as cianofíceas e Bicudo e Menezes (25) para as clorofíceas e euglenofíceas. A riqueza (R) total do fitoplâncton foi considerada como o número total de táxons encontrados em cada unidade amostral.

A determinação da densidade fitoplanctônica foi feita de acordo com o método de sedimentação de Utermöhl (26). Organismos unicelulares, filamentos e colônias foram considerados como indivíduos e a contagem cobriu toda a área da câmara com os resultados expressos em organismos por litro (org/L).

A abundância relativa foi obtida com a contagem dos 100 primeiros organismos presentes em uma alíquota retirada da amostra qualitativa, sendo expressa em percentagem 
(\%) e para sua interpretação foi utilizada a escala segundo Lobo e Leighton (27). O critério adotado para calcular a frequiência de ocorrência foi o mesmo utilizado por Mateucci e Colma (28).

Os índices de diversidade específica foram determinados utilizando-se a matriz de dados da densidade de cada táxon. Os índices foram calculados na mesma base logarítmica (base 2) para que pudessem ser comparados, sendo a diversidade determinada segundo Shannon e Weaver (29) e a equitabilidade segundo Pielou (30). Após determinação da diversidade, os valores obtidos foram enquadrados na classificação de Valentin et al. (31)

O teste de Mann-Whitney foi utilizado para verificar se existe diferença significativa entre os parâmetros abióticos ( $\mathrm{pH}, \mathrm{OD}$, Salinidade e Temperatura) coletados na superfície e próximo ao fundo e para mostrar se parâmetros abióticos (pH, OD, Salinidade, Temperatura, Transparência) diferiram entre estações de amostragem (E1 e E2). O teste não paramétrico de Kruskal-Wallis foi executado para verificar se os parâmetros abióticos diferiram significativamente ao longo do cultivo. Foi feita uma associação entre as variáveis abióticas e entre as variáveis abióticas e os grupos do microfitoplâncton, considerando que esta comunidade responde muito rapidamente às alterações físicas e químicas do meio aquático. Uma associação entre as variáveis abióticas e a clorofila "a" também foi realizada. Estas associações foram feitas por meio de correlação linear simples com coeficiente de Pearson (r) considerando o $\mathrm{p} \leq 0,05$ para que as correlações fossem consideradas significativas. As análises descritas foram realizadas através do programa BioStat 5.0.

\section{RESULTADOS}

O pH teve mínima de 8,1 e máxima de 9,4 na Estação 02, com discreta variação ao longo do cultivo. $\mathrm{O}$ oxigênio dissolvido teve um comportamento bastante irregular ao longo do cultivo, sendo o menor valor registrado $4,2 \mathrm{mg} / \mathrm{L}$ na Estação 01 e o maior, $15,5 \mathrm{mg} / \mathrm{L}$ na Estação 02. Nas estações de coleta, a salinidade se manteve estável (30 ppm) até a $3^{\text {a }}$ data de coleta, sofrendo um declínio e em seguida, voltando a subir. A menor temperatura registrada na Estação 01 foi de $31,5^{\circ} \mathrm{C}$ no primeiro dia de coleta e a maior, $35,0{ }^{\circ} \mathrm{C}$ na Estação 02 (última coleta). A transparência apresentou máxima de $55 \mathrm{~cm}$ na primeira coleta, diminuindo para $17 \mathrm{~cm}$ no fim do ciclo de cultivo. A Tabela 1 apresenta as médias das variáveis físicoquímicas estudadas.

Tabela 1. Médias obtidas entre superfície e fundo das variáveis físico-químicas mensuradas em viveiro de criação de L. vannamei durante um ciclo de cultivo

\begin{tabular}{lcccccccccc}
\hline Coleta/ & \multicolumn{2}{c}{$\mathbf{p H}$} & \multicolumn{2}{c}{ OD $(\mathbf{m g} / \mathbf{L})$} & \multicolumn{2}{c}{ TE $\left({ }^{\mathbf{o}} \mathbf{C}\right)$} & \multicolumn{2}{c}{ S $(\mathbf{p p m})$} & \multicolumn{2}{c}{ TR $(\mathbf{c m})$} \\
\cline { 2 - 11 } Estação & $\mathbf{1}$ & $\mathbf{2}$ & $\mathbf{1}$ & $\mathbf{2}$ & $\mathbf{1}$ & $\mathbf{2}$ & $\mathbf{1}$ & $\mathbf{2}$ & $\mathbf{1}$ & $\mathbf{2}$ \\
\cline { 2 - 11 } 20/set & 8,1 & 8,1 & 9,6 & 8,5 & 31,7 & 33,0 & 30,0 & 30,0 & 55 & 45 \\
07/out & 8,1 & 8,3 & 4,2 & 4,9 & 31,7 & 32,3 & 30,0 & 30,0 & 33 & 37 \\
22/out & 9,2 & 9,4 & 13,5 & 15,6 & 32,6 & 32,7 & 30,0 & 30,0 & 17 & 20 \\
05/nov & 9,1 & 9,1 & 14,7 & 13,6 & 33,5 & 33,5 & 26,5 & 27,0 & 18 & 20 \\
20/nov & 8,3 & 8,1 & 5,2 & 5,3 & 31,5 & 33,5 & 27,5 & 27,5 & 25 & 25 \\
05/dez & 9,0 & 8,9 & 9,8 & 9,6 & 33,0 & 35,0 & 30,0 & 28,5 & 20 & 20 \\
\hline
\end{tabular}

OD - Oxigênio Dissolvido; TE - Temperatura; TR - Transparência

Durante o estudo, a precipitação mínima foi de $0,0 \mathrm{~mm}$ em novembro e a máxima de $122,8 \mathrm{~mm}$ em dezembro (Quadro 1), ressaltando que o período amostrado trata-se da estação seca no município.

Quadro I. Precipitação Pluviométrica (mm) no município de Curuçá no ano de 2008. 


\begin{tabular}{|l|l|l|l|l|l|l|l|l|l|l|l|l|}
\hline \multirow{2}{*}{ PP (mm) / Meses } & Jan & Fev & Mar & Abr & Mai & Jun & Jul & Ago & Set & Out & Nov & Dez \\
\cline { 2 - 11 } & 471,7 & 279,1 & 820,7 & 490,3 & 311,7 & 0,0 & 0,0 & 26,9 & 17,1 & 17,5 & 0,0 & 122,8 \\
\hline
\end{tabular}

PP - Precipitação Pluviométrica. Fonte: Agência Nacional das Águas, 2009

A comunidade fitoplanctônica esteve representada por 95 táxons infraespecíficos e específicos, distribuídos entre as Divisões: Bacillariophyta (69 táxons), Chlorophyta com 14 táxons, Pyrrophycophyta (5 táxons), Cyanophyta (6 táxons) e Euglenophyta (1 táxons), como observado na Figura 2.

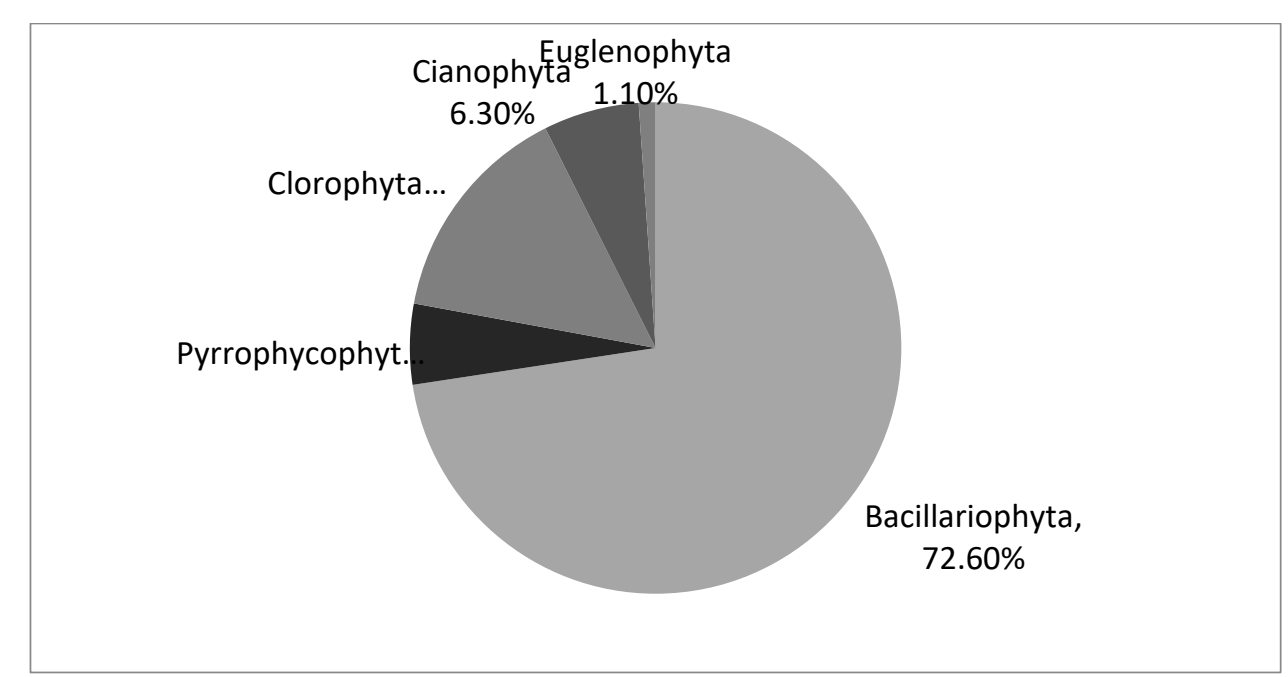

Figura 2. Distribuição percentual das divisões na composição do microfitoplâncton em viveiro de criação de $L$. vannamei durante um ciclo de cultivo.

Na estação 01, a clorofila $a$ aumentou ao longo do cultivo, atingindo um máximo de $471,34 \mathrm{mg} / \mathrm{m}^{3}$ dia $20 / 11$ e em seguida, sofreu uma queda, $387,64 \mathrm{mg} / \mathrm{m}^{3}$. Na estação 02 , o comportamento desta variável foi semelhante, mas os valores registrados foram menores, sendo o máximo de $250,8 \mathrm{mg} / \mathrm{m}^{3}$ na $4^{\circ}$ coleta (dia 05/11). A amostra do dia 05/Nov da Estação 01 foi perdida, devido um pequeno acidente de carro.

Em termos de densidade total, não houve uma tendência uniforme de aumento ou decréscimo nos seus valores ao longo do ciclo investigado, sendo o menor valor registrado de 3.600 org/L na Estação 01 e o maior de 104.400 org/L na Estação 02. Em relação à densidade específica, observou-se que Navicula, Pleurosigma e Nitzschia foram os gêneros mais abundantes, sendo os principais responsáveis pela dominância da divisão Bacillariophyta nas duas estações de coleta. Quanto à abundância relativa, a divisão Bacillariophyta foi um grupo que variou de abundante a dominante (Figura 3), com porcentagem muito acima dos demais grupos. A divisão Pyrrophycophyta teve maior importância no início do cultivo ( $2^{\circ}$ coleta), atingindo 32\% de abundância relativa na Estação 01 e 27\% na $1^{\circ}$ coleta da Estação 02. 


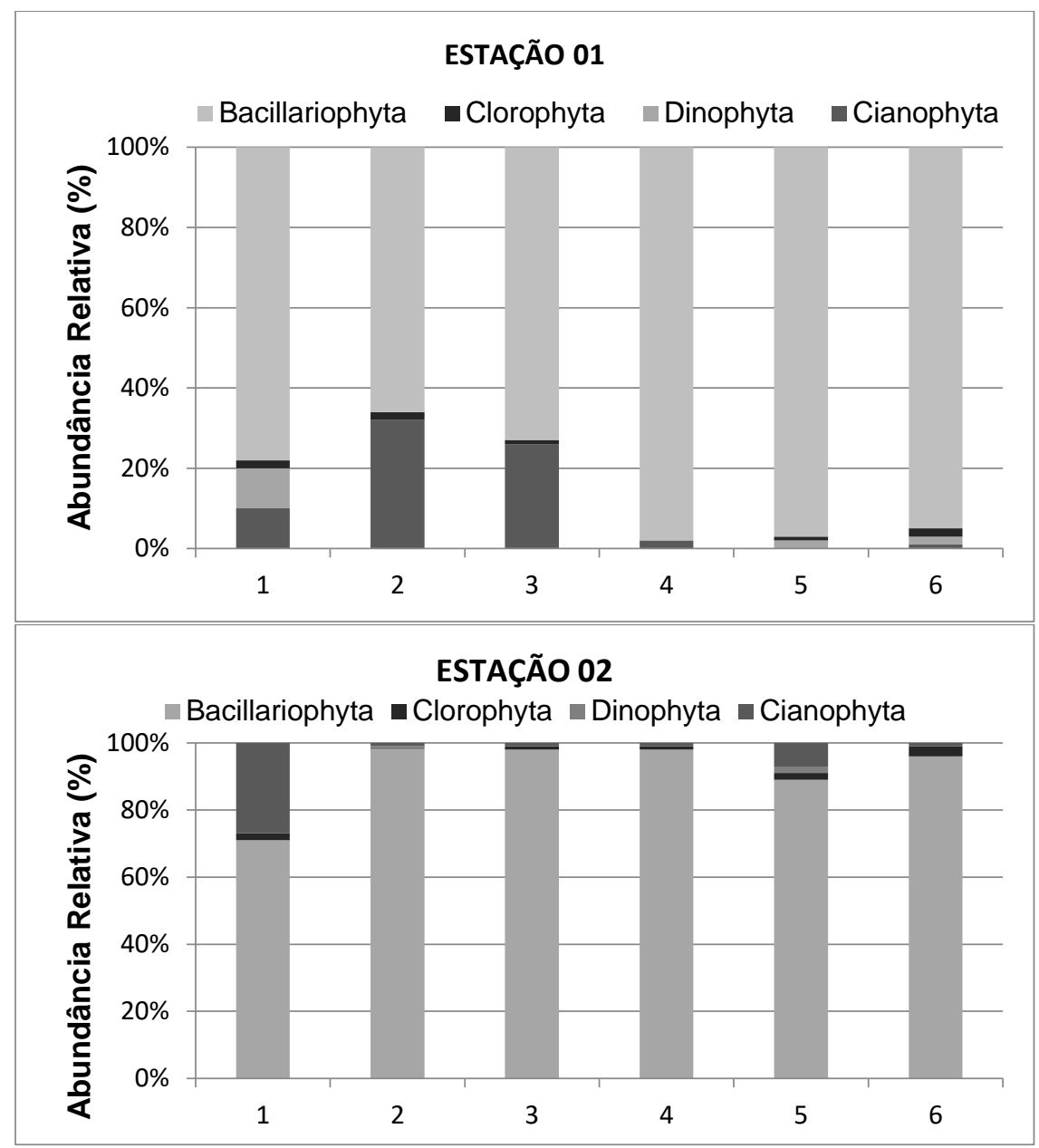

Figura 3. Abundância relativa microfitoplâncton em viveiro de criação de L. vannamei nas seis coletas realizadas durante um ciclo de cultivo.

As espécies que ocorreram em todas as amostras foram: Amphiprora alata, Aulacoseira granulata, Coscinodiscus spp, Cyclotella spp, Fragilaria sp, Melosira arctica, Navicula spp, Nistchia spp, Odontella mobiliensis, Oscillatoria sp, Pleurosigma sp, Polimyxus coronalis, Spyrogyra sp e Thalassiosira spp. A diversidade média ( $\left.\mathrm{H}^{\prime}\right)$ nas estações de coleta teve um máximo de 2,0 bits/ind e um mínimo de 0,96 bits/ind. Em relação à equitabilidade média $(\mathrm{J}$ ') nas estações estudadas, a máxima foi de 0,76 e a mínima de 0,28.

A análise de Kruskal-Wallis apresentou diferença significativa ao longo do cultivo para os seguintes parâmetros abióticos: $\mathrm{pH}(\mathrm{H}=19,36 ; \mathrm{p}=0,0016)$; oxigênio dissolvido $(\mathrm{H}=$ 20,63; $\mathrm{p}=0,0010)$; e transparência $(\mathrm{H}=21,29 ; \mathrm{p}=0,0007)$. Somente temperatura e salinidade não diferiram significativamente ao nível de 0,05 durante a criação de $L$. vannamei.

A Tabela 2 mostra que não houve diferença significativa em nenhuma das situações testadas, aceitando-se as hipóteses que a mensuração feita na superfície é a mesma próxima ao fundo; a média obtida é igual nas estações de coleta; a densidade total obtida é igual nas estações de coleta.

O teste de correlação linear realizado entre os parâmetros abióticos mostrou que as variáveis selecionadas pelo teste como significativas foram: transparência com o pH $(\mathrm{r}=$ $0,83 ; \mathrm{p}=0,04)$ e oxigênio dissolvido com o $\mathrm{pH}(\mathrm{r}=0,83 ; \mathrm{p}=0,04)$.

O teste de correlação linear realizado entre as variáveis abióticas ( $\mathrm{pH}, \mathrm{OD}$, Salinidade, Transparência e Temperatura) e os grupos do microfitoplâncton mostrou que somente uma variável foi selecionada pelo teste como significativa: a densidade de dinoflagelados com a transparência $(\mathrm{r}=0,91 ; \mathrm{p}=0,01)$. 
O teste de correlação linear executado entre os parâmetros abióticos e a clorofila "a" mostrou que somente uma variável foi selecionada pelo teste como significativa: a transparência, que se correlacionou negativamente com a clorofila " $a$ " $(r=-0,86 ; p=0,02)$.

Tabela 2 - Valores de p resultantes dos testes de hipóteses

\begin{tabular}{|c|c|c|c|c|}
\hline Variável em teste & Teste & $\begin{array}{c}\text { Estatística do } \\
\text { teste }\end{array}$ & p valor & Análise \\
\hline Estação 01: Superf. x Fundo & Mann-Whitney & & & \\
\hline $\mathrm{pH}$ & ، & 0,5604 & 0,5752 & NS \\
\hline OD & ، & 0,6405 & 0,5218 & NS \\
\hline Salinidade & ، & 0,0000 & 1,0000 & NS \\
\hline Temperatura & ، & 1,6813 & 0,0927 & NS \\
\hline Estação 02: Superf. x Fundo & Mann-Whitney & & & \\
\hline $\mathrm{pH}$ & , & 0,1601 & 0,8728 & NS \\
\hline OD & ، & 0,6405 & 0,5218 & NS \\
\hline Salinidade & ، & 1,0408 & 0,2980 & NS \\
\hline Temperatura & ، & 1,3611 & 0,1735 & NS \\
\hline Média Est. 01 x Est. 02 & Mann-Whitney & & & \\
\hline $\mathrm{pH}$ & ، & 0,2402 & 0,8102 & NS \\
\hline OD & ، & 0,0000 & 1,0000 & NS \\
\hline Salinidade & ، & 0,2402 & 0,8102 & NS \\
\hline Temperatura & ، & 1,6013 & 0,1093 & NS \\
\hline Transparência & ، & 0,4804 & 0,6310 & NS \\
\hline Dens. Fito Est. 01 x Est. 02 & Mann-Whitney & 1,1219 & 0,2623 & NS \\
\hline
\end{tabular}

NS - Não Significativo

\section{DISCUSSÃO}

De acordo com Nunes (32), no cultivo de camarões marinhos a temperatura da água está intimamente associada à temperatura do ar, oscilando para cima e para baixo de forma proporcional. $\mathrm{O}$ autor afirma que a faixa ideal de temperatura para a espécie L. vannamei é entre 26 e $33{ }^{\circ} \mathrm{C}$. Martins (4) estudando um ciclo semi-intensivo de cultivo de L. vannamei no Ceará registrou máxima de $25,9^{\circ} \mathrm{C}$ e Chelappa et al. (9) em estudo semelhante no Rio Grande do Norte registraram $30,7{ }^{\circ} \mathrm{C}$ como a temperatura mais elevada, enquanto que no presente estudo a maior temperatura amostrada foi de $35^{\circ} \mathrm{C}$, o que poderia causar estresse nos animas confinados.

$\mathrm{Na}$ estação seca, é comum observar altas temperaturas e altas salinidades e provavelmente elas coexistem devido à redução de água doce, através de um fluxo menor proveniente dos rios e da chuva. Neste trabalho, as temperaturas foram mais elevadas que as registradas por Pereira et al. (12) que estudando o estuário do rio Curuçá no período seco registrou temperatura máxima de $30,5^{\circ} \mathrm{C}$. Isso revelou que a temperatura no ambiente de cultivo é mais elevada que em ambiente natural, provavelmente devido à baixa profundidade do viveiro e à elevada insolação na região. A temperatura não variou significativamente ao longo do cultivo $(\mathrm{p}>0,05)$.

Em relação ao oxigênio dissolvido (OD), sabe-se que é o mais importante e crítico na qualidade da água, para o L. vannamei, índices superiores a $3 \mathrm{mg} / \mathrm{L}$ propiciam um melhor desenvolvimento (33). No viveiro estudado, mesmo sem sistema de aeração artificial não foi detectado nenhum valor abaixo de $4 \mathrm{mg} / \mathrm{L}$, apresentando valores mais elevados que os registrados por Pereira et al. (12) no Estuário do rio Curuçá (máximo de 8,2 mg/l de OD), 
provavelmente devido a atividade fotossintética intensa durante as coletas (período da manhã) e maior concentração espacial dos organismos fitoplanctônicos. Fonseca (6) registrou a máxima concentração de 10,8 mg/l, enquanto que Martins (4) obteve valores entre 0,3 e 5,9 $\mathrm{mg} / \mathrm{l}$, ambos estudos no estado do Ceará.

Os maiores valores de OD foram observados nos dias 22/out e 05/nov, período em que a água de cultivo esteve mais turva, sendo registradas as menores transparências, o que permite inferir a relação direta entre o fitoplâncton e a produção de OD, promovendo incremento dos níveis de OD a partir de intensa atividade fotossintética. Porém, altas densidades fitoplanctônicas podem alterar o desenvolvimento do camarão devido à depleção do OD durante a noite (34), período em que o fitoplâncton realiza o processo de respiração juntamente com os demais organismos presentes no ambiente de cultivo.

$\mathrm{O} \mathrm{OD}$ correlacionou-se positivamente com o pH $(\mathrm{r}=0,83)$, revelando que à medida que aumenta uma das variáveis, a outra também aumenta. Noriega et al. (35) estudando o estuário de Barra das Jangadas (PE) também observaram uma correlação positiva entre pH e oxigênio dissolvido.

Quanto ao $\mathrm{pH}$, apesar de existirem poucas informações sobre os efeitos do $\mathrm{pH}$ no desenvolvimento dos camarões (1), algumas mensurações estiveram acima de 9,0, que estaria acima da faixa ideal para o cultivo da espécie segundo Hernadez e Nunes (36), que é entre 8,1 e 9,0. Chelappa et al. (9) estudando durante um ciclo de cultivo viveiros de engorda de $L$. vannamei no Rio Grande do Norte obtiveram dados de $\mathrm{pH}$ variando de 8,1 a 8,2. Pereira et al. (12) registrou os valores de $\mathrm{pH}$ sempre na faixa caracteristicamente alcalina, variando de 7,2 a 8,0 no estuário do rio Curuçá.

Foi detectada diferença significativa ao longo do cultivo para o $\mathrm{pH}$ e $\mathrm{OD}(\mathrm{p}<0,05)$, o que pode estar ligado à maior atividade fotossintética, que ao promover a assimilação de $\mathrm{CO}_{2}$ pelo processo, acarreta a elevação do $\mathrm{pH}$ (37) que está correlacionado positivamente ao OD.

O comportamento da salinidade neste estudo foi semelhante ao observado por Cavalcanti (5) em um ciclo de cultivo semi-intensivo de L. vannamei na Paraíba. Os altos valores de salinidade registrados são justificados pelo período de estudo estar compreendido na época menos chuvosa na região, que vai de julho a dezembro (18), na qual a cunha salina de água do mar aproxima-se do continente, penetrando no estuário e aumentando os teores de salinidade do rio. A salinidade não variou significativamente ao longo do cultivo ( $p>0,05)$. Na pesquisa, a máxima salinidade observada foi de $30 \mathrm{ppm}$, estando dentro do recomendado por Boyd (33), que é de o a 40 ppm.

Em relação à transparência da água, Boyd (33) indica como uma faixa adequada entre 0,30 e $0,45 \mathrm{~m}$. Foi possível observar que a transparência diminuiu ao longo do cultivo, semelhantemente aos resultados encontrados por Cavalcanti (5) em dois ciclos de cultivo em viveiros na Paraíba e por Fonseca (6) em um viveiro no Ceará. A média de transparência registrada para as estações de coleta 01 e 02 foram 28 e $27,8 \mathrm{~cm}$, respectivamente, sendo inferiores à média obtida por Martins (4), que foi de $50 \mathrm{~cm}$ em viveiros de cultivo semiintensivo localizados no Estado do Ceará.

A transparência correlacionou-se negativamente com o $\mathrm{pH}(\mathrm{r}=-0,83)$, isto porque em geral a medida da transparência permite inferir que quanto mais turva e escura a água estiver, mais rica em fitoplâncton estará. Essa comunidade ao promover maior atividade fotossintética, vai assimilar durante processo $\mathrm{CO}_{2}$, o que acarreta elevação do $\mathrm{pH}$. A transparência variou significativamente ao longo do tempo $(\mathrm{p}<0,05)$, revelando a influência do cultivo nesta variável, seja pelo maior aporte de ração, maior incremento do volume de dejetos excretados pelos animais e/ou ressuspensão do sedimento em dias mais ventilados.

Não foi detectada diferença significativa $(\mathrm{p}>0,05)$ para nenhum parâmetro abiótico entre superfície e próximo ao fundo e nem entre as estações de amostragem, revelando um ambiente saudável, homogêneo e sem o fenômeno da estratificação.

Silva RS, Souza ASL, Palheta GDA, Costa MSM, Melo NFAC. Diversidade e biomassa fitoplanctônica em viveiro de carcinicultura marinha no estado do Pará. Vet. e Zootec. 2018 jun.; 25(2): 142-154. 
Sendo a clorofila um dos principais pigmentos responsáveis pela fotossíntese, o conhecimento de sua concentração pode dar indicações da biomassa do fitoplâncton. Nos últimos anos, tem se tornado cada vez mais frequente a utilização da concentração de clorofila para expressar a biomassa fitoplanctônica (37).

As concentrações de clorofila $a$ foram sofrendo aumento ao longo do cultivo, assim como o observado por Fernandes (8) que registrou a máxima concentração de $381 \mathrm{mg} / \mathrm{m} 3$, no período de seca, coincidindo com o período estudado neste trabalho. Considerando que não foi realizado nenhum procedimento de fertilização de manutenção durante o cultivo, os valores de clorofila $a$ deveriam estar abaixo do que foi registrado. Provavelmente a não renovação de água no sistema de cultivo adotado, favoreceu concentrações muito elevadas de clorofila $a$.

Quanto à riqueza, neste estudo foram identificados 95 taxa, número superior ao observado por Melo et al. (10) com 28 taxa e Casé et al. (38) com 51 espécies, porém inferior ao inventariado por Fonseca (6), que foi de 225.

Em relação à diversidade média, Valentin et al. (31) classifica as estações de coleta variando de baixa a muito baixa, o que pode ser um indicativo de um sistema desequilibrado com diminuição da qualidade da água ou apenas uma característica de ambientes voltados à aquicultura. Quanto à equitabilidade, a classificação de Valetin et al. (31) revela que as amostras se encontraram melhor distribuídas no início e no fim do cultivo, já que os maiores valores ocorreram nesse período (mais próximos de um). Portanto, apesar de o sistema estudado ter baixa diversidade de espécies, elas estavam bem distribuídas.

Quanto á representatividade de cada Divisão, Bacillariophyta respondeu a $73 \%$, bem próximo ao registrado por Melo et al. (10) que foi de $71,4 \%$, enquanto que Fonseca (6) registrou predominância de diatomáceas (aproximadamente 100\%), mas somente no início do ciclo de cultivo, pois depois houve um maior predomínio de cianofíceas. Neste estudo a divisão Bacillariophyta foi o grupo dominante, confirmando a alta diversidade de diatomáceas em águas estuarinas. Segundo Patil e Gislerod (39), as diatomáceas são um importante recurso alimentar para camarões, tendo uma composição química rica em vitaminas e ácidos graxos. Já na pesquisa de Melo et al. (10), a Classe Cianoficeae foi predominante.

Neste trabalho Nitzschia, Navicula e Pleurosigma foram os principais gêneros responsáveis para a dominância das diatomáceas. Chelappa et al. (9) e Casé et al. (38) também identificaram as diatomáceas como dominantes em viveiros de carcinicultura marinha. Na região de Curuçá, a ação dos ventos é contínua e a profundidade do viveiro é pequena, permitindo uma ressuspensão do sedimento através do revolvimento do fundo, o que explicaria a maior abundância das diatomáceas bentônicas Pleurosigma, Navicula e Nitzschia neste estudo. Dam et al. (40) citam Nitzschia e Navicula como gêneros de grande representatividade, indicadores de águas ricas em nutrientes.

Segundo Smayda (41), as diatomáceas destacam-se como os principais organismos do microfitoplâncton estuarino e, na maioria dos casos, elas constituem a maior percentagem das microalgas presentes. Em estuários, a ocorrência e distribuição dos vários grupos de algas fitoplanctônicas são influenciadas pela salinidade, sendo as clorofíceas, cianofíceas e euglenofíceas relativamente mais importantes em locais com baixos teores salinos, tornandose bem mais adaptadas em ambientes de água doce. Isso explica a menor representatividade desses grupos no estudo (41). O domínio de diatomáceas em viveiros de carcinicultura marinha é uma condição satisfatória, pois comparando com outros tipos de algas, as bacillariophytas são um melhor alimento para o camarão $(1,7)$.

As densidades fitoplanctônicas não apresentaram uma tendência uniforme de aumento ou decréscimo nos seus valores ao longo do ciclo investigado nas estações de coleta, assim como o observado por Pereira Neto (7) e Fonseca (6). A densidade de dinoflagelados correlacionou-se positivamente com a transparência $(r=0,91)$, revelando que esse grupo do 
microfitoplâncton não é bem adaptado em ambientes turvos, se desenvolvendo melhor em ambientes mais transparentes. Isto é confirmado por Pollingher (42) que afirma que a classe Dinophyceae tem seu desenvolvimento favorecido por grande disponibilidade de luz e elevadas temperaturas. Em carcinicultura, os dinoflagelados são muitas vezes prejudiciais aos camarões cultivados, assim é o caso das "marés vermelhas" de algumas espécies de Peridinium, o qual pode resultar na perda da produção (7).

\section{CONCLUSÃO}

Concluiu-se que fertilizações de manutenção não se mostraram necessárias, pois os restos de ração e fezes dos animais foram suficientes na liberação de nutrientes para o desenvolvimento da comunidade fitoplanctônica, fato observado a partir das concentrações de clorofila $a$ e densidades ao longo do cultivo; A divisão Bacillariophyta que foi o grupo mais importante tanto em termos de riqueza quanto de densidade, podendo representar uma excelente alternativa como alimento natural no cultivo; $\mathrm{O} \mathrm{pH}$, oxigênio dissolvido e transparência devem ser constantemente monitorados, devido variação que obtiveram ao longo do cultivo; $O$ fenômeno da estratificação não foi detectado, o que torna a profundidade de 1,5m uma recomendação em viveiros de carcinicultura do nordeste paraense.

\section{AGRADECIMENTOS}

À CAPES, pela bolsa de mestrado da primeira autora. Aos estagiários do Laboratório de Ecologia Aquática e Aqüicultura Tropical da Universidade Federal Rural da Amazônia e ao proprietário e funcionários da Fazenda São Paulo.

\section{REFERÊNCIAS}

1. Boyd CE. Water quality in ponds for aquaculture. Alabama: Agricultural Experimental Station, Arlburn University; 1990.

2. Boyd CE. Potencial of sodium nitrate to improve environmental conditions in aquaculture ponds. World Aquac. 1995;26:38-40.

3. Allan GL, Moriarty DJW, Maguire GB. Effects of pond preparation and feeding rate on production of Peanaeus monodon Fabricius, water quality, bacteria and benthos in model farming. Aquaculture. 1995;130:329-49.

4. Martins PCC. Influência das condições ambientais e técnicas de produção sobre a incidência de enfermidades no cultivo de camarão marinho, Litopenaeus vannamei, no Estado do Ceará [tese]. São Carlos: Universidade Federal de São Carlos; 2003.

5. Cavalcanti LB. Variações das condições hidrológicas e da clorofila a associadas ao cultivo do camarão marinho Litopenaeus vannamei (Boone, 1931), na região estuarina do rio Paraíba do Norte (Paraíba - Brasil) [tese]. Recife: Universidade Federal de Pernambuco; 2003.

6. Fonseca RS. Dinâmica da comunidade fitoplanctônica em um viveiro de engorda de camarão marinho (Litopenaeus vannamei) no estado do Ceará [dissertação]. Fortaleza: Universidade Federal do Ceará; 2006.

7. Pereira Neto JB, Dantas DMM, Gálvez AO, Brito LO. Avaliação das comunidades planctônica e bentônica de microalgas em viveiros de camarão (Litopenaeus vannamei). Bol Inst Pesca. 2008;34(4):543-51. 
8. Fernandes D, Oliveira L, Pereira L, Gomes G, Freire G. Caracterização de amônia, nitrato, nitrito, fosfato (orto) dissolvido e clorofila "a" em uma fazenda de cultivo de camarão. Rev Geol. 2007;20(1):99-117.

9. Chelappa NT, Lima AKA, Câmara FRA. Riqueza de microalgas em viveiros de cultivo orgânico de camarão em Tibau do Sul, Rio Grande do Norte. Rev Bras Biocienc. 2007;5(2):120-2.

10. Melo MP, Carvalheiro JMO, Cordeiro TA, Queiroz AR, Prado JP, Borges IF. Phytoplanktonic composition of three cultivation systems used in Litopenaeus vannamei (BOONE, 1931) marine shrimp farms. Acta Sci Biol Sci. 2010;32(3):223-8.

11. Santana DS, Paiva RS, Melo NFAC. Diatomáceas cêntricas da região entre-marés da Praia de Ajuruteua (Bragança-Pará). Bol Mus Para Emílio Goeldi. 2006;1(3):149-56.

12. Pereira CTC, Giarrizzo T, Jesus AJS, Martinelli JM. Caracterização do efluente de cultivo de Litopenaeus vannamei no estuário do Rio Curuçá (PA). In: Barroso GF, Poersch LHS, Cavalli RO. Sistemas de cultivos aquícolas na zona costeira do Brasil: recursos, tecnologias e aspectos ambientais e sócio-econômicos. 1a ed. Rio de Janeiro: Museu Nacional; 2007. p.291-302.

13. Sousa EB, Costa VB, Pereira LCC, Costa RAAM. Microfitoplâncton de águas costeiras amazônicas: Ilha Canela (Bragança, PA, Brasil). Acta Bot Bras. 2008;22:626-36.

14. Sousa EB, Costa VB, Pereira LCC, Costa RAAM. Variação temporal do fitoplâncton e dos parâmetros hidrológicos da zona de arrebentação da Ilha Canela (Bragança-ParáBrasil). Acta Bot Bras. 2009;23:1084-95.

15. Santana DS, Paiva RS, Pereira LCC, Costa RM. Microphytoplankton of the Marapanim Estuary (Pará, Northern Brazil). Trop Oceanogr. 2010;38(2):152-63.

16. Costa VB, Sousa EB, Pinheiro SCC, Pereira LCC, Costa RAAM. Effects of high energy coastal environment on the structure and dynamics of phytoplankton communities (Brazilian Amazon littoral). J Coast Res. 2011;64:354-8.

17. Matos JB, Silva NIS, Pereira LCC, Costa RM. Caracterização quali-quantitativa do fitoplâncton da zona de arrebentação de uma praia amazônica. Acta Bot Bras. 2012;26:979-90.

18. Instituto de Desenvolvimento Economico, Social e Ambiental do Pará. Estatística Municipal - Curuçá. Belém: IDESP; 2014.

19. Melo JSC. Água e construção de viveiros na piscicultura. Lavras: UFLA/FAEPE; 1999.

20. United Nations Educational, Scientific, and Cultural Organization. Determination of photosynthethic pigments in sea water. Monographys on Oceanography Methodology, 1. Paris: Unesco; 1966. 
21. Parsons TR, Strickland JDH. Discussion of spectrophotometric determination of arine plankton pigments, with revised equations of as certaninig clorophyll a and caratenoids. $\mathbf{J}$ Mar Res. 1963;21(3):155-63.

22. Round FE, Crawford RM, Mann DG. The diatoms biology and morphology of the genera. Cambridge: Cambridge University; 1990.

23. Steindinger KA, Tangen K. Dinoflagelates. In: Tomas CR. Identifying marine diatoms and dinoflagelates. San Diego: Academic Press; 1997. p.387-584.

24. Desikachary TS. Cyanophyta. New Delhi: Council of Agricultura Researcer; 1959.

25. Bicudo EM, Menezes M. Gêneros de algas de águas continentais do Brasil. São Carlos: Rima; 2005. (Chave para identificação e descrições).

26. Utermöhl H. Zur Vervollkommung der quantitativen Phytoplankton-Methodik. 9a ed. Stuttgart: Schweizerbart; 1958. p.1-38.

27. Lobo E, Leighton G. Estructuras comunitárias de lãs fitocenosis planctônicas de los sistemas de desembocaduras de rios y esteros de la zona central de Chile. Rev Biol Mar. 1986;22:1-29.

28. Matteucci SD, Colma A. Metodologia para el estudio de la vegetacion. Washington: Secretaria General de La Organización de los Estados Americanos; 1982.

29. Shannon CE, Weaver W. The mathematical theory of communication. Urbana: University Illinois Press; 1949.

30. Pielou EC. The measurement of diversity in different types of biological collection. $\mathbf{J}$ Theoret Biol. 1966;13:131-44.

31. Valentin JL, Macedo-Saidah FE, Tenenbaum DR, Silva NML. A diversidade específica para a análise das sucessões fitoplanctônicas. Aplicação ao ecossistema da ressurgência de Cabo Frio (RJ). Nerítica. 1991;6(1-2):7-26.

32. Nunes AJP. O impacto da temperatura no cultivo de camarões marinhos. Rev ABCC. 2002;4(1):43-8.

33. Boyd CE. Manejo da qualidade da água na aquicultura e no cultivo de camarão marinho. 1a ed. Recife: Associação Brasileira de Criadores de Camarão - ABCC; 2000.

34. Alonso-Rodriguez R, Páez-Osuna, F. Nutrients, phytoplankton and harmful algal blooms in shrimp ponds: a review with special reference to the situation in the Gulf of California. Aquaculture. 2003;219(1-4):317-36.

35. Noriega CD, Muniz K, Feitosa FAN, Flores-Montes MJ, Grego CKS, Soares GSS, et al. Distribuição espacial da biomassa fitoplanctônica e sua relação com os sais nutrientes, no sistema estuarino de Barra das Jangadas (Pernambuco - Brasil). Arq Cienc Mar. 2005;38:5-18. 
36. Hernàdez JZ, Nunes AJP. Biossegurança no cultivo de camarão marinho: qualidade da água e fatores ambientais. Rev ABCC. 2003;3(2):55-9.

37. Esteves FA. Fundamentos de Limnologia. 2a ed. Rio de Janeiro: Interciências/ FINEP; 1998.

38. Casé M, Leça EE, Leitão S, Moraes Júnior AT. Plankton community as an indicator of water quality in tropical shrimp culture ponds. Mar Pollut Bull. 2008;56(7):1343-52.

39. Patil V, Gislerød HR. The importance of omega-3 fatty acids in diet. Curr Sci. 2006;90(7):908-9.

40. Dam H, Mertens A, Sinkeldam JA. Coded checklist and ecological indicator values of freshwaters diatoms from the Netherlands. Neth J Aquat Ecol. 1994;28(1):117-33.

41. Smayda TJ. The phytoplankton of estuaries. In: Ketchum BH. Estuaries and enclosed seas. Ecosystems of the World, 26. Amsterdam: Elsevier; 1983. p.65-102.

42. Pollingher U. Freshwater armored dinoflagellates: growth, reproduction strategies and population dynamics. In: Sandgreen CD. Growth and reproductive strategies of freshwater phytoplankton. Cambridge: Cambridge University Press; 1988. p.134-74.

Recebido em:

Aceito em: 